\title{
Towards Optimal Task Distribution on Computer Clusters with Intel MIC Coprocessors
}

\author{
Chenggang Lai, Miaoqing Huang \\ Department of Computer Science and Computer Engineering \\ University of Arkansas \\ \{c1004,mqhuang\}@uark.edu
}

\author{
Genlang Chen \\ Ningbo Institute of Technology \\ Zhejiang University \\ cgl@zju.edu.cn
}

\begin{abstract}
Computer clusters with coprocessors/accelerators are typically leveraged to parallelize applications for reducing computation time. Given $N$ parallel tasks and $M$ processing cores, the typical strategy is to statically distribute those $N$ tasks among $M$ cores so that each core receives $\frac{N}{M}$ tasks. However, for many sophisticated applications, the processing times of $N$ tasks may vary. In other words, some tasks will take longer time than others. The static distribution will cause the cores with light tasks to wait for the cores with the heavy tasks, resulting in an imbalance in task distribution and the nonminimal overall processing time for the application. In this work we apply dynamic task distribution. All the unfinished tasks form a task pool. Once a core finishes a task, it will request a new task from the task pool. Through this manner, all cores will be kept busy in the whole computation process. We apply two additional optimization techniques to further improve the performance of applications on clusters with Intel MIC coprocessors. First, we design hybrid implementations to distribute tasks to both the CPUs and MICs. Second, we apply multiple-level parallelism technique to realize the concurrency among the $N$ tasks as well as the concurrency in each task. We use sparse coding as a case study to demonstrate the advantages of our approach. Sparse coding is a class of unsupervised methods for learning sets of over-complete bases to represent data efficiently. The aim of sparse coding is to find a set of basis vectors such that an input vector can be represented as a linear combination of these basis vectors. The results show that the dynamic task distribution can improve the performance by $25 \%$ compared with the static one. Further, the hybrid mode implementation involving both the host CPUs and the MICs can outperform the basic offload mode implementation by $\mathbf{4 0 \%}$.
\end{abstract}

\section{INTRODUCTION}

Computer clusters with coprocessors/accelerators are typically leveraged to parallelize applications for reducing computation time. Given $N$ parallel tasks and $M$ processing cores, the typical strategy is to statically distribute those $N$ tasks among $M$ cores so that each core receives $\frac{N}{M}$ tasks. For example, given 1,024 tasks and 4 processing cores, core_0 takes tasks $0-255$, core_1 takes tasks $256-511$, etc. This kind of static task distribution is fine for applications in which each task requires almost the same amount of processing time. However, for many sophisticated applications such as sparse coding, it will be shown later that those parallel tasks require different amounts of processing times. In such case, the workloads distributed to those cores will become uneven. In the previous example, if tasks 0-255 are quite heavy and tasks 256-511 are relatively light, then core_1 will take less time to process its tasks and then stays idle while waiting for core_0 to finish.
In order to resolve the imbalance of workload among those cores, it is better to distribute those tasks to the participating cores dynamically. In order to achieve the dynamic task distribution, all tasks will form a task pool. Every time a core will request an available task from the pool. Once it finishes the current task, it will go to request a new task from the pool. In this way, all cores will work together to finish the tasks in the pool and spend more or less the same amount of time on data processing.

Computer clusters typically contain both powerful multicore CPUs and massively parallel manycore coprocessors/accelerators. Therefore it is desired to distribute workload to both the host CPUs and the coprocessors to take advantages of both types of processors. Our results show that the hybrid implementation in which the workload is evenly divided into CPUs and MICs can almost double the performance compared with the implementation in which only the MICs are used.

The rest of the paper is organized as follows. In Section II we introduce the sparse coding benchmark and various parallel implementations based on MIC. The experimental results and evaluation using the AUVs seafloor image data set are presented in Section III. We conclude this work in Section IV.

\section{The Benchmark: Sparse CODING}

\section{A. Problem statement}

Sparse coding is an algorithm for constructing succinct representations of input vectors such as images using the basis vectors in a dictionary [1]. Given a set of $n$ 2D images, each image can be represented by a $1 \mathrm{D}$ array $\overrightarrow{x^{(i)}}, i=1 \ldots n$. For example, if an image contains $32 \times 32$ pixels, the corresponding $\overrightarrow{x^{(i)}}$ will consist of 1,024 elements. The aim of sparse coding is to find a set of basis vectors $\overrightarrow{d^{(j)}}(j=1 \ldots k)$ such that we can represent an input vector $\overrightarrow{x^{(i)}}$ as a linear combination of these basis vectors, $\overrightarrow{x^{(i)}}=\sum_{j=1}^{k} a_{j}^{(i)} \overrightarrow{d^{(j)}}$, in which each $a_{j}^{(i)}$ is a scalar coefficient. If we use $D$ and $\overrightarrow{a^{(i)}}$ to represent $\left.\overrightarrow{\left[d^{(1)}\right.}, \overrightarrow{d^{(2)}}, \ldots \overrightarrow{d^{(k)}}\right]$ and $\left[a_{1}^{(i)}, a_{2}^{(i)}, \ldots a_{k}^{(i)}\right]^{T}$, respectively, we can re-write the equation as $\overrightarrow{x^{(i)}}=D \overrightarrow{a^{(i)}}$. Further, if we use $X$ and $A$ to represent $\left[\overrightarrow{x^{(1)}}, \overrightarrow{x^{(2)}}, \ldots \overrightarrow{x^{(n)}}\right]$ and $\left[\overrightarrow{a^{(1)}}, \overrightarrow{a^{(2)}}, \ldots \overrightarrow{a^{(n)}}\right]$, respectively, we want to represent $X$ as $X=D A$. Each column of $D$ (i.e., $\overrightarrow{d^{(j)}}$ ) is regarded as a basis in the directory. Each column of $A$ (i.e., $\left.\overrightarrow{a^{(i)}}\right)$ is the sparse representation of the corresponding input vector $\overrightarrow{x^{(i)}}$ according to the dictionary. 


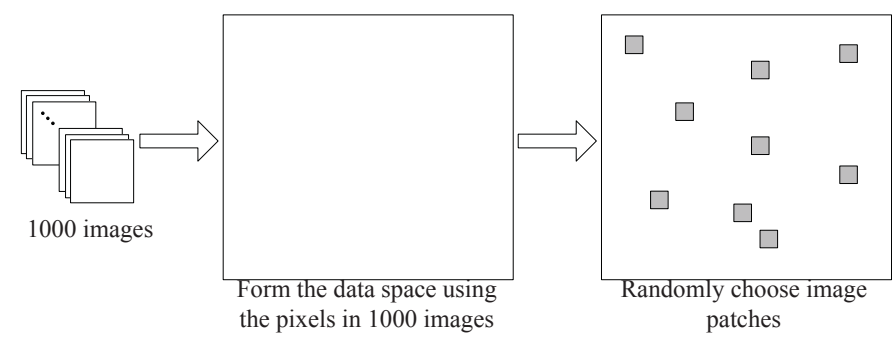

Fig. 1. The process to form the source data space and randomly choose several data sets.

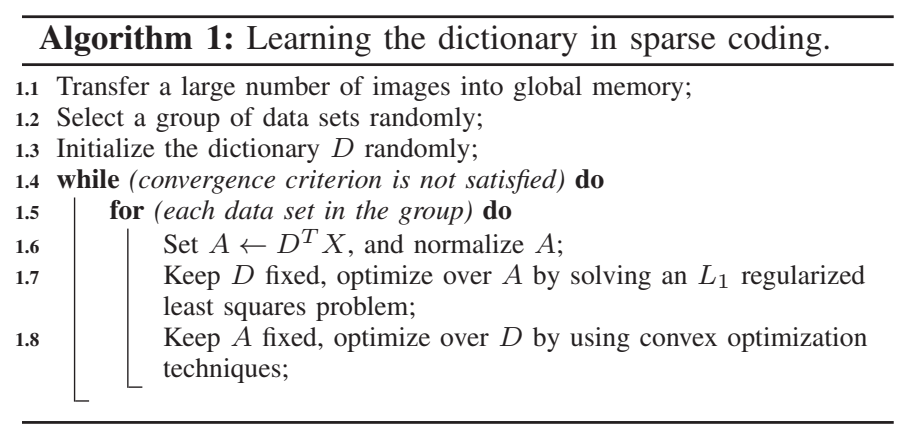

Most components of $\overrightarrow{a^{(i)}}$ should be zero's. The basic idea of sparse coding is defined as finding two matrices $D$ and $A$ and aims to solve the following optimization problem:

$$
\min _{A, D} \sum_{i=1}^{n}\left(\left\|\overrightarrow{x^{(i)}}-\sum_{j=1}^{k} a_{j}^{(i)} \overrightarrow{d^{(j)}}\right\|^{2}+\lambda \sum_{j=1}^{k}\left|a_{j}^{(i)}\right|\right) .
$$

It has been shown that the optimization problem is not jointly convex in both $A$ and $D$, but it is convex in either $A$ or $D$ if the other one is kept fixed. An alternating minimization algorithm has bee proposed in [2], as shown in Algorithm 1. When facing large numbers of images and dictionary bases, the step of optimizing over $A$ is particularly time consuming since it involves an uncertain objective function.

We conducted a simple test to check the computation times spent on each step. The original data set is comprised of 14 dive missions conducted by the AUV Sirius off the southeast coast of Tasmania in October 2008 [3], [4]. It contains over 100,000 stereo pairs of images. Marine scientists used the CPCe software package [5] to label 50 random points on each image with different class labels, such as biological species (including coral, algae and others), abiotic elements (sand, gravel, rock, shell, etc.), and other unknown data types. In this test, we chose $10001,024 \times 1,024$ images from the original data set. Then we randomly chose 5 sets of image patches. Each set contains 1,024 patches, each of which is of $32 \times 32$. The process to form the source data space and choose the data sets is illustrated in Figure 1. Once the 5 sets of image patches were generated, we applied the optimization on them using Algorithm 1. In this simple test, we only carried out 3 iterations of the while loop in Algorithm 1. This implementation was a single-thread implementation written in

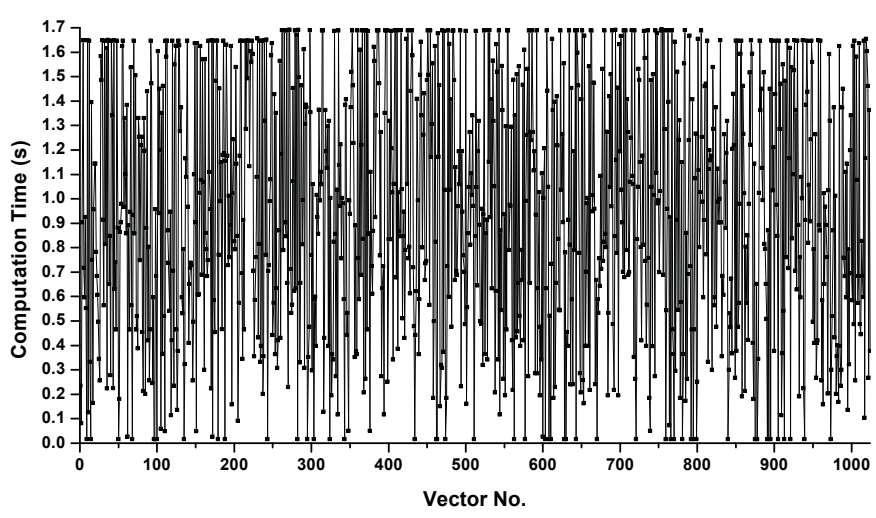

Fig. 2. The computation times of 1,024 coefficient vectors in the sequential implementation on CPU.

$\mathrm{C}$ and executed on an Intel Xeon E5606 2.13-GHz CPU. The step of optimizing over $A$ took $14,811.29$ seconds while the total time was $15,027.34$ seconds.

There are two levels of parallelism we can take advantages of. In the first level (level-1) of parallelism, it can be found that all the columns of $A$, i.e., $\overrightarrow{a^{(i)}}$ 's, can be optimized independently. However, the computation times of those coefficient vectors vary quite significantly. Figure 2 shows the computation times of 1,024 vectors in our benchmark. The times are in the range of $[0.016 s, 1.694 s]$. A static distribution of those 1,024 vectors will result in an imbalance of computation among participating processing cores. In the second level (level-2) of parallelism, the updating of 1,024 components in each $\overrightarrow{a^{(i)}}$ can be parallelized as well. In our implementation, the updating of one $\overrightarrow{a^{(i)}}$ is a combination of sequential stages and parallel stages. Those parallel stages can be carried out by multiple cores.

\section{B. Parallelization using $M I C$}

The parallel sparse coding can be implemented with the following execution modes:

- Native-1: i.e., the native model. In this implementation, the MPI process is directly executed on each MIC core, each of which only executes a single-thread MPI process. For sparse coding, only the level-1 parallelism is realized.

- Native-2: On top of Native-1 execution mode, we launch 4 threads in each MPI process using OpenMP. Therefore, this mode will provide two levels of parallelism: parallel MPI processes for the level-1 parallelism and OpenMP threads in each MPI process for the level-2 parallelism.

- Offload: In this mode, the MPI processes are hosted by the CPU cores, which offload the computation including data to the MIC processors. The host MPI process on CPU issues multiple threads to the MIC card using OpenMP so that each thread works on one or more coefficient vectors depending on the number of participating MIC cards. If several coefficient vectors are assigned to one thread, they will be processed in a sequence. Then we apply a second level of parallelism, i.e., spawning multiple threads in each thread. Given the 240 threads 
that can be physically executed in parallel on the Xeon Phi 5110P device, various configurations can be applied. If we use $M$ and $N$ to represent the level-1 and level-2 threads, respectively, different combinations of $(M, N)$ can be adopted subject to $M \times N \leqslant 240$. This mode can be further categorized into two sub-modes, i.e., Offload$\mathbf{S}$ for static task distribution and Offload-D for dynamic task distribution.

- Hybrid: In this mode, both CPUs and MICs are allocated for data processing. First the workload is distributed to CPUs through MPI. Then a host CPU will offload part of the workload to a MIC card using OpenMP. On the host CPU, we also use OpenMP to spawn multiple threads for parallel processing. The peak performance of an Intel MIC 5110P is around 2,022 GFLOP/s, which is about 4 times the performance of a single thread on the host CPU (i.e., Intel Xeon E5-2670). Therefore we run 4 threads on the host CPU and evenly divide the workload between a host CPU and its corresponding MIC processor. The hybrid mode also has two variants, Hybrid-S and Hybrid-D.

At this moment, we could not find a solution to achieve the dynamic task distribution in MPI. Therefore, for the native mode, the task distribution among all MPI processes/ranks is static. For the offload mode, the first level task distribution to the host CPUs is through MPI. Therefore, it is still static. Once the host CPU tries to offload tasks to the MIC coprocessor, it can be either static or dynamic.

\section{EXPERIMENT AND EVALUATION}

In order to evaluate the performance of the parallel methods on Intel MIC based computer clusters, a series of experiments were carried out on a large and high-quality AUV (Autonomous Underwater Vehicle) data set. In this section we present the experiment platform and the results.

\section{A. Experiment platform}

We conduct our experiments on the NSF sponsored Beacon supercomputer [6]. The Beacon system (a Cray CS300-AC Cluster Supercomputer) offers the access to 48 compute nodes and $6 \mathrm{I} / \mathrm{O}$ nodes joined by FDR InfiniBand interconnect, which provides a $56 \mathrm{~Gb} / \mathrm{s}$ bi-directional bandwidth. Each compute node is equipped with 2 Intel Xeon E5-2670 8-core 2.6-GHz processors, 4 Intel Xeon Phi (MIC) coprocessors 5110P, 256 GB of RAM, and $960 \mathrm{~GB}$ of SSD storage. Each I/O node provides access to an additional $4.8 \mathrm{~TB}$ of SSD storage. Each Xeon Phi 5110P coprocessor contains $601.053-\mathrm{GHz}$ MIC cores and 8 GB GDDR5 on-board memory. The compiler used in this work is Intel 64 Compiler XE, Version 14.0.0.080 Build 20130728.

\section{B. Results and discussion}

We conduct the experiments to use multiple MIC processors to demonstrate the scalability and performance of the learning dictionary process, which is the most time-consuming part in

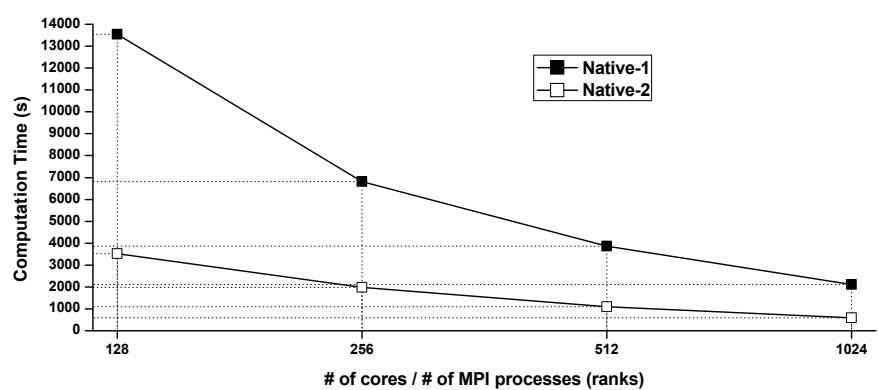

Fig. 3. Performance scalability under the native execution modes. 60 MPI processes (ranks) are scheduled to one MIC card.

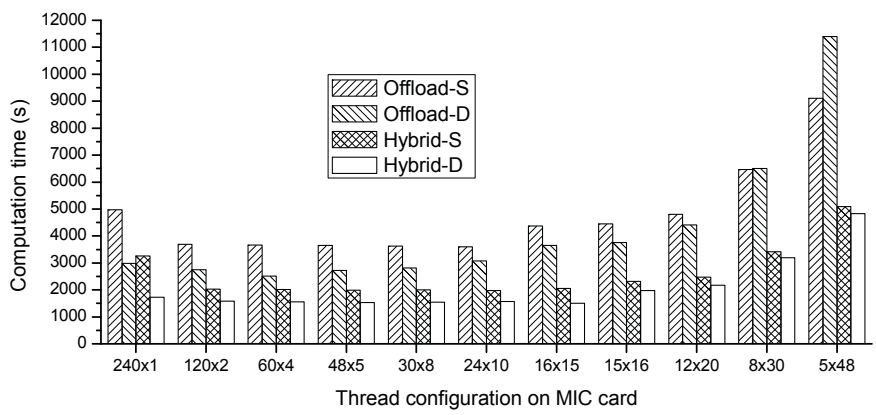

Fig. 4. Performance comparison between static distribution and dynamic distribution as well as between offload mode and hybrid mode on a single MIC card. $M \times N: M$ is the number of threads offloaded to a MIC card for realizing the level-1 parallelism, each thread further spawns $N$ threads to achieve the level-2 parallelism. 4 threads are created on the host CPU for the level-1 parallelism.

sparse coding methods. In our experiments, we mainly evaluate the following three aspects: the performance scalability of the native modes, and the performance improvement due to dynamic task distribution on a single MIC card as well as on multiple MIC cards.

1) Performance scalability of the native modes: In order to show the strong scalability of the parallel implementations, the problem size is fixed for each implementation while the number of participating cores is increased. For the native modes, each MIC will host one MPI process. We schedule 60 MPI processes to a MIC card.

From Figure 3 we can see that both implementations in native mode keep the strong scalability, i.e., the computation time halves when the number of processing cores doubles. Because the Native-2 implementation uses 4 times of threads as the Native-1 implementation, its performance is approximately 4 times better than the Native- 1 mode.

2) Performance improvement of dynamic task distribution on a single MIC card: We first show the benefit of the dynamic task scheduling compared with the static task scheduling on a single MIC card. Because there are multiple combinations of the number of the threads scheduled to a MIC card and the number of threads spawned by each thread, we list 11 options in Figure 4. When there are many level-1 threads, the advantage of dynamic distribution is very evident. For example, when there are more than 30 level- 1 threads, the performance improvement is more than $25 \%$. When the number of level-1 threads decrease, the difference between the 


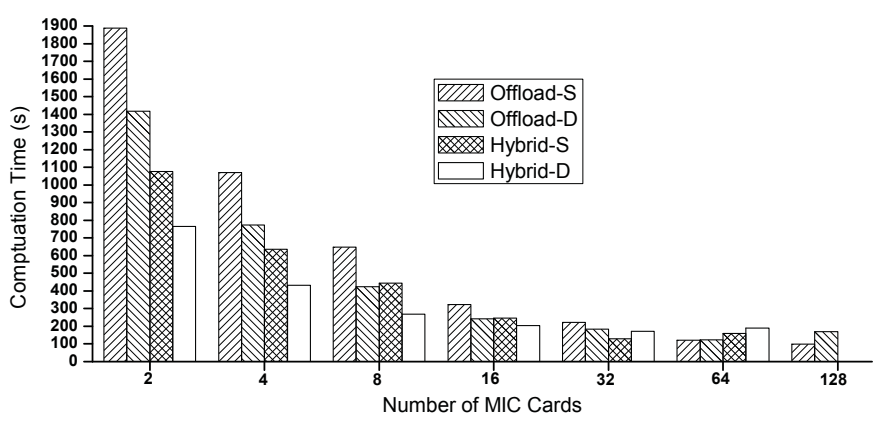

Fig. 5. Performance comparison between static distribution and dynamic distribution as well as between offload mode and hybrid mode on multiple cards. For hybrid modes, 4 CPU threads are scheduled for one MIC card. Two MIC cards are hosted by one CPU, which will execute 8 threads. Therefore, the ratio between the number of host CPUs and the number of MIC cards is $1: 2$

TABLE I

THREAD CONFIGURATION IN MULTIPLE-MIC IMPLEMENTATIONS.

\begin{tabular}{c||c|c|c|c}
\hline Number of MIC cards & Offload-S & Offload-D & Hybrid-S & Hybrid-D \\
\hline \hline 2 & $24 \times 10$ & $60 \times 4$ & $60 \times 4$ & $60 \times 4$ \\
\hline 4 & $24 \times 10$ & $60 \times 4$ & $60 \times 4$ & $60 \times 4$ \\
\hline 8 & $24 \times 10$ & $60 \times 4$ & $60 \times 4$ & $60 \times 4$ \\
\hline 16 & $24 \times 10$ & $60 \times 4$ & $60 \times 4$ & $16 \times 15$ \\
\hline 32 & $24 \times 10$ & $30 \times 8$ & $16 \times 15$ & $16 \times 15$ \\
\hline 64 & $16 \times 15$ & $16 \times 15$ & $16 \times 15$ & $16 \times 15$ \\
\hline 128 & $16 \times 15$ & $16 \times 15$ & - & - \\
\hline
\end{tabular}

static distribution and dynamic one diminishes. There are two reasons. First each level-1 thread will receive many tasks in both distributions. This will reduce the imbalance of workload among those threads. Second, because the computation of one coefficient vector consists of both sequential stages and parallel stages, the sequential stages will become a limiting factor for performance improvement when a large number of level-2 threads are spawned. More level-2 threads will not further improve the performance once the number of level-2 threads reaches a certain point.

Figure 4 also shows that the hybrid mode can improve the performance by more than $40 \%$ compared with the offload mode. In this implementation, we artificially distribute the total workload evenly between the host CPU and the MIC coprocessor. Therefore we only issue 4 threads on the CPU for pairing with one MIC card. The host CPU, Xeon E5-2670, contains 8 cores and can execute 16 threads physically. In theory, we can schedule more work to the host CPU. This reminds us that it is very important to schedule workload to both the host CPUs and the coprocessors for achieving the best performance. The ratio of the workload between these types of processors needs to be carefully assigned for realizing the balance. Between the two variants of the hybrid mode, the comparison is similar to the case of the offload mode. The Hybrid-D mode can improve the performance by $25 \%$ compared with the Hybrid-S mode when the number of level1 threads is abundant.

3) Performance improvement of dynamic task distribution on multiple MIC cards: We also conduct the experiment by using multiple MIC cards. The thread configurations for various implementation modes are different and listed in Table I. The performance saving trend is very similar to the one-MIC-card case. As shown in Figure 5, for both offload mode and hybrid mode, the dynamic task distribution is able to improve the performance by more than $25 \%$ when the number of MIC cards is less than 16 . When more MIC cards are added, the communication overhead will become dominant. Both hybrid implementations are able to outperform their offload counterparts by around $40 \%$ when there are significant amount work scheduled to each MIC card and CPU (i.e., the number of MIC cards is less than 16). Beyond that, the performance gain due to hybrid implementation starts diminishing due to the introduced communication overhead.

\section{CONClusions}

In this work we conduct a detailed study regarding the performance and scalability of various parallel execution modes on computer clusters with Intel MIC coprocessors. The tasks in an application are typically distributed to multiple nodes for parallel processing. However, for sophisticated applications such as sparse coding, those parallel tasks may require different processing times. A static task distribution among working threads may introduce an imbalance of workload among them. In order to improve the overall performance, we leverage the dynamic task distribution so that a thread will request a new task from a task pool once it finishes the processing of the current task. Experiment results show that this technique can improve the performance by $25 \%$. In addition, we try to leverage the processing power of the host CPU by implementing the sparse coding algorithm in a hybrid mode. The results show that the hybrid implementation is able to further improve the performance by $40 \%$.

\section{ACKNOWLEDGMENTS}

This work is supported in part by the Special Scientific Research Fund for Public Welfare Industry of the Chinese State Ocean Administration (Grant No.: 201305026), by NSF (Grant No.: CNS-1219062), and by NASA (Grant No.: 122012EPSCoR-0026).

\section{REFERENCES}

[1] B. A. Olshausen et al., "Emergence of simple-cell receptive field properties by learning a sparse code for natural images," Nature, vol. 381, no. 6583, pp. 607-609, 1996.

[2] H. Lee, A. Battle, R. Raina, and A. Y. Ng, "Efficient sparse coding algorithms," in Advances in neural information processing systems, 2006, pp. 801-808.

[3] M. Bewley, B. Douillard, N. Nourani-Vatani, A. Friedman, O. Pizarro, and S. Williams, "Automated species detection: An experimental approach to kelp detection from sea-floor auv images," in Proceedings of Australasian Conference on Robotics and Automation, Victoria University of Wellington, New Zealand, 2012.

[4] N. Barrett, L. Meyer, N. Hill, and P. Walsh, "Methods for the processing and scoring of auv digital imagery from south eastern tasmania," 2011.

[5] K. E. Kohler and S. M. Gill, "Coral point count with excel extensions (cpce): A visual basic program for the determination of coral and substrate coverage using random point count methodology," Computers \& Geosciences, vol. 32, no. 9, pp. 1259-1269, 2006.

[6] http://www.jics.tennessee.edu/aace/beacon. 